\title{
An Exploration of Surface Integrity Remanufacturing for Aeroengine Components
}

\begin{abstract}
Surface integrity is the major factor impacting on the operation quality, service life and reliability of the aeroengine components. The surface integrity of aeroengine component is damaged by the failures such as crack, deformation, oxidation, corrosion, erosion, and microstructural degeneration. It adopts advanced remanufacturing technologies to restore or improve the surface integrity and regenerate these high value parts. This paper firstly puts forward the concept, namely surface integrity remanufacturing for aeroengine components, and its connotation. The key remanufacturing technologies have been developed to repair the components with surface damages. Ultimately, some application examples of surface integrity remanufacturing technologies as well as their effects in aeroengine maintenance are introduced. The discarded components have been reused and their service lives have been extended and their reliability has been increased by implementing surface integrity remanufacturing. It has realized "The Repaired Components Outpacing the New Ones", material saving, energy saving, and emission reduction.
\end{abstract}

Keywords: aeroengine component, surface integrity, remanufacturing, surface integrity remanufacturing

\section{Introduction}

The concept of surface integrity (SI) was originally introduced by Dr. Field and Dr. Kahles of Metcut Research Associates at the 1964 Tripartite Technical Coordinating Symposium (Field \& Kahles, 1971). SI can be defined as the topographical, mechanical, chemical and metallurgical

Manuscript received January 15, 2016; accepted May 1, 2016

Qiao Xiang (凶)

Air Engine Corporation of China, Beijing 100072, China; Chengdu Holy Industry \& Commerce Co. Ltd. (Group), Chengdu 610044, China Email: xiangq@cae.cn

Yong He, Ting-hong Hou

Chengdu Holy Industry \& Commerce Co. Ltd. (Group), Chengdu 610044, China characteristics of a manufactured surface and their relationships to functional performance (ANSI-B211.1, 1986; Griffiths, 2001). The aerospace industry is among the first to consider the surface integrity of their components, since the consequences of a breakage are always dramatic from a human and economical point of view. Aeroengine components are subjected to severe conditions: Stress, temperature, oxidation, corrosion and other hostile environments. Surface condition has an ever-increasing influence on the performance of the components. Many previous failure analyses of aeroengine components show that severe failures, produced by fatigue, creep, stress, erosion and corrosion cracking, always start on the surface or near the surface of components. The origin for failures depends on the surface quality largely. Surface integrity reveals the influence of surface properties and condition upon which materials are likely to perform. Therefore, surface integrity has a significant impact on the working performance, durability and reliability of aeroengine components. In 1971, the surface integrity system of aerospace materials was established by American Aerospace Materials Laboratory based on systematic process experiments and life evaluation methods to improve the surface integrity of the materials and extend the working life of the components.

Various types of physical damages can occur due to the formation of cracks, voids and worn surfaces, under aggressive environments during the operation of aeroengine components. Because the cost of the components is relatively high, it is typically more desirable to repair these damaged components than to replace them. Remanufacturing is one of the most efficient ways of recycling worn parts because it consumes only a fraction of energy, cost, and material required for new parts. The surface integrity remanufacturing is an important part of the aeroengine component remanufacturing. The surface integrity of the components can be restored or improved by using remanufacturing technologies.

In this paper, the concept and connotation of surface integrity remanufacturing is put forward firstly and then the key technologies of surface integrity remanufacturing and the aeroengine remanufacturing system are introduced and 
finally the application examples and effects of surface integrity remanufacturing are demonstrated.

\section{Concept and connotation}

\subsection{Surface integrity}

Surface integrity was defined by Field and Kahles (1971) as "the unimpaired or enhanced surface condition or properties of a material resulting from a controlled manufacturing process". According to the original concept of surface integrity, it can be extended to six different groups of key factors: Visual, dimensional, residual stress, tribological, metallurgical, and other factors as described in Figure 1. The six groups of key factors are also applicable to aeroengine components. When dealing with manufacturing issues, it is important to keep in mind that all the parameters involved in the finishing process have a direct influence on the surface integrity of the part. There are three main objectives of surface integrity in aerospace industry: Minimum weight or small section, high degree of safety and economical competition. The combination of these three objectives makes this job very difficult. In such a context, the surface integrity of their components is of primary importance.

From a general view, surface integrity is the sum of all of the elements that describe all the conditions existing on or at the surface of the finished part. Surface integrity has two aspects. As shown in Figure 2, the first is surface topography which describes the roughness, lay or texture of the outermost layer of the part and the second is surface metallurgy which describes the nature of the altered layers below the surface with respect to the base or matrix material. It is the assessment of the impact of manufacturing processes on the properties of the part material. Surface topography is concerned with the geometry of the outermost layer of the workpiece, its texture and its interface with the environment. Surface metallurgy is concerned primarily with the host of effects a process has below the visible surface. The subsurface characteristics occur in various layers or zones. The subsurface altered material zones (AMZ) can be as simple as a stressed condition different from that in the body of the material or as complex as a grain structure change interlaced with inter-granular attack (IGA). Changes can be caused by chemical, thermal, electrical, or mechanical energy and affect both the physical and the metallurgical properties of the material.

In a broad sense, the concern of surface integrity is for surface quality. The benefits that can accrue from surface integrity control are as follows:

- A better understanding of the process and process control limits and better quality control;

- Machinability data enhanced by surface integrity limits and better definition of manufacturing leeway;

- A reduction in scrap or rework incidents;

- Cost avoidance by use of surface integrity practices only where required;

- Guidance to advanced process design or application.

\subsection{Surface integrity remanufacturing}

Remanufacturing is the process of returning a used product (nonfunctional, discarded, or traded in) to at least the performance specification of an original product (Biller, 2011). It is one form of product end-of-life strategies, often

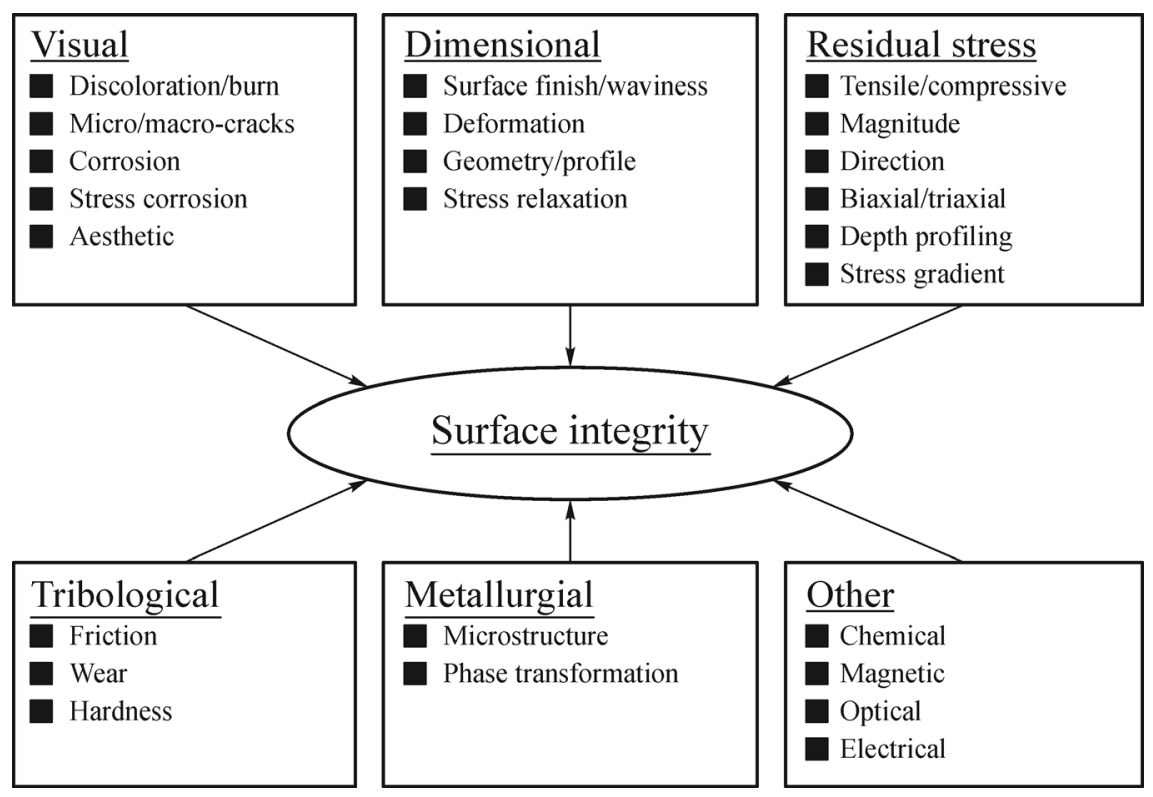

Figure 1. The six different groups of key factors defining the surface integrity of a finished material. 


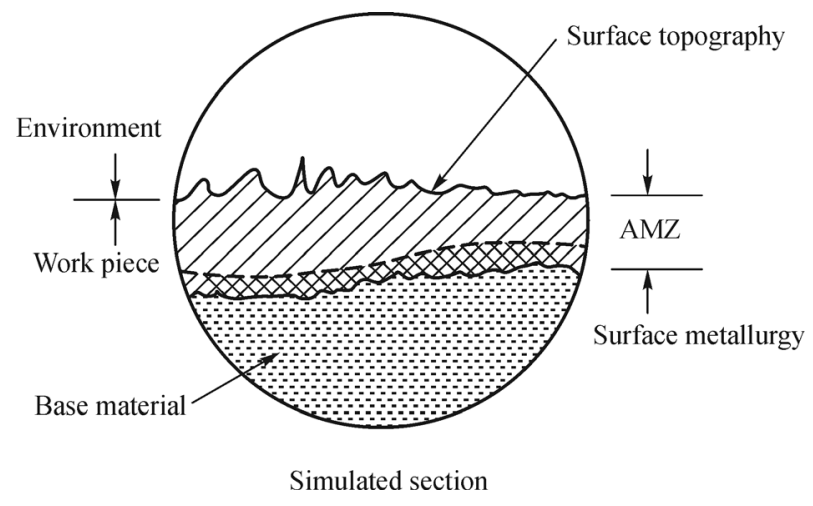

Figure 2. The concept of surface integrity (AMZ: Altered material zone).

called 6R: Reduce, reuse, recycle, recover, redesign, and remanufacture, aiming for resource efficiency (Axinte \& Dewes, 2002). Moreover, it is an advanced stage during the process of maintenance and development and a new form of advanced manufacturing. Its position and function in the product life cycle is shown in Figure 3. Remanufacturing differs from recycling in that it recovers both the material value and the functional value of a used product. In view of its environmental and economic benefits, remanufacturing is gaining significant ground in the global scenario.

Surface integrity concerns not only the topological (geometric) aspects of surfaces but rather the whole assemblage of their physical, mechanical, metallurgical, and chemical characteristics. Its objective is to assure the required service properties of surfaces in part manufacturing because many manufacturing operations directly affect these properties.

In the author's opinion, the requirements for surface integrity vary with the stages in the life cycle of the component. The initial surface integrity of the component is determined by its design requirement and machining process. The surface integrity is gradually degraded or damaged in working environments with the passage of service time. The main problem to be resolved is the repair and improvement of later surface integrity in remanufacturing. Therefore, the surface integrity remanufacturing can be defined as the engineering activities of repairing the surface defects and damages by using remanufacturing technologies to restore or improve the required surface integrity. From a technical point of view, surface integrity remanufacturing refers to the integration of technologies (innovation, universality and scientificalness) and processes (stability, applicability and adaptability) to improve their working performance and extend their service life. It plays an important role in aeroengine components remanufacturing, and the surface quality and working performance of the components should not be less than those of the new ones after surface integrity remanufacturing.

The ever-increasing strength capabilities of the new aerospace materials have been accompanied by an increase in sensitivity to machining processing variables. The concern for surface integrity remanufacturing is the reflection of concern for component integrity and can be summarized as follows:

- Thin and complex sections are more prevalent;

- More sensitive and difficult alloys are being employed;

- Higher stress/strain levels are usual;

- Designs are closer to material limits and capabilities;

- Reliability requirements are more stringent and durability requirements are increasing;

- Awareness that there is a significant depth of impact of processes on materials is increasing.

\section{Key technologies of surface integrity remanufacturing}

Although aerospace materials are continuously updated and the new alloys are more reliable than ever before, they still experience severe environment like high temperature and highly oxidized corrosion during the service, where the component damages are unavoidable. Theoretically, the damaged components need to be replaced because of the surface defects such as cracks, erosion, corrosion and deformation. However, the high cost of replacement directs the owners' preference into the component repair technology over simple replacement of damaged parts. Therefore,

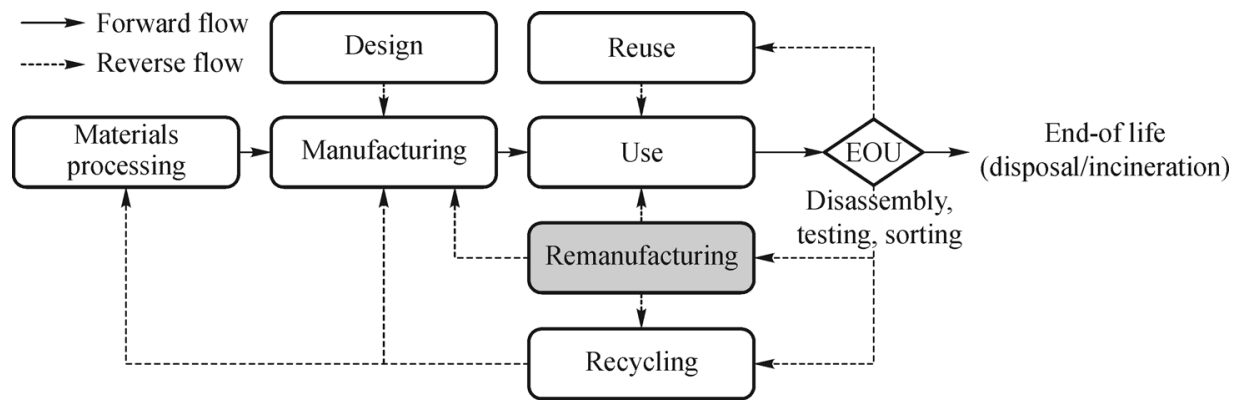

Figure 3. The position and function of remanufacturing in the product life cycle (EOU: End of use). 
how to develop more efficient and economical repair schemes has drawn people's interests as a big challenge.

In the late 1970s, a five-year cooperative research project in European Community was initiated to carry out the fundamental research on welding, diffusion brazing and coating technologies for aeroengine component repair in the Netherlands. At the same time, the United States, Sweden, Germany and England had invested heavily in repair technologies development for hot-section components and obtained a group of valuable practical repair technologies including vacuum brazing, wide-gap brazing, laser welding and electron beam welding. These repair technologies can be regarded as the origin of surface integrity remanufacturing technology. Through the development of last several decades, many new technologies such as laser cladding, thermal spraying, micro-plasma welding, surface modification, rejuvenation heat treatment and powder metallurgy have come out (Ellison, Lowden, \& Liburdi, 1993). All of these aforementioned technologies aim to solve the common problems of surface integrity and are used to repair the components with the surface defects such as crack, wear, erosion, corrosion and deformation. These technologies can be classified into three broad categories: Welding and brazing, heat treatment, coating and plating, and surface strengthening. Some key technologies and processes of surface integrity remanufacturing are introduced as follows.

\subsection{Welding and brazing}

Modern welding technologies such as laser, gas tungsten arc and plasma-transferred arc welding and brazing processes such as transient liquid phase bonding, diffusion bonding, vacuum brazing and wide gap brazing are used to return service exposed components to a serviceable condition and their surface integrity.

\subsubsection{Welding}

Welding is widely used in the repair of cracking, rubbing, corrosion, and erosion damage on the components. It is found that gas tungsten arc welding (GTAW), laser beam welding (LBW), electron beam welding (EBW) and plasma-transferred arc welding (PTAW) are increasingly used in remanufacturing processes. Welding repair has its limitation in repairing high strength superalloys, particularly those containing higher $\gamma^{\prime}$ primer formers $(\mathrm{Al}+\mathrm{Ti})$ since they are highly susceptible to hot cracking in the weld metal and the heat affected zone (HAZ), and strain-age cracking (or post weld heat treatment) in HAZ. Poor weldability of most high strength precipitation hardened nickel-base superalloys requires pre- and post-heating or the use of more compliant weld filler materials with lower strength than the parent metals. The typical practical examples of welding repair are the tip repairs of high- pressure turbine blade with tip cracks, low-pressure turbine blade with worn shroud Z interlocks and compressor blade with tip erosion and foreign object damage, and the patch repair of combustion chamber with crack and corrosion.

It should be noted that it is practically impossible to make welds in high strength, precipitation hardened alloys without some level of micro-cracking. Therefore, in addition to the limits on weld location, limits are also placed on the size, shape and orientation of acceptable defects. Most problems with welding of alloys relate to the following two effects: One is that high internal stresses (approaching the yield strength) are set up locally during welding due to the intense local heating of the welding heating source, the other is that metallurgical transformations take place in the base metal adjacent to the weld (HAZ), resulting in undesirable mechanical properties.

Laser cladding is a deposition welding process in which a layer of powder is deposited on the substrate material, and the two materials are fused by metallurgical bonding through the action of a laser beam. It is a relatively new technology used in aviation maintenance filed. It is clear that laser cladding presents considerable advantages for the repair of nickel-based superalloys over traditional welding. It produces little distortion and resulted in high quality deposits. In general, the exceptional properties of laser cladding depend critically on the proportions of the alloying elements. In order to obtain the best performance, it is essential to avoid dilution of the depositing material by the substrate, as hardness is inversely proportional to dilution.

\subsubsection{Brazing}

Due to the limitations of welding, brazing was developed as an alternative remanufacturing technique and is widely used for the repair of stationary components such as vanes, stators and combustion liners. The typical practical examples of brazing repair are the repair of nozzle vane craze cracks and the repair of superalloy castings casting defects.

According to gap width to be repaired, the brazing can be divided into two broad categories: conventional (narrow gap) brazing and wide gap brazing. Conventional brazing involves the use of materials with a melting point lower than the material being repaired. The parts are heated in a furnace to a temperature above the melting temperature of the braze alloy and it flows into the joint or defect by capillary forces. Cruces to this behaviour are the joint gap which must be small to generate capillary forces and surface cleanliness which is required for wetting to occur. It is the capillary forces that limit the application of conventional brazing and the brazing clearances that range from 0.03 to $0.08 \mathrm{~mm}$ results in superior capillary action and good joint strength.

Wide gap brazing (WGB) exploits the mechanism of 
diffusion brazing, where the strong reaction between braze alloy and base metal is achieved via melting, dissolution, and diffusion, in bridging larger clearances. Over the past three decades, many wide gap and modified wide gap processes have been developed and employed in the repair of hot section components such as activated diffusion healing (ADH) by General Electric (GE) (Demo \& Ferrigno, 1992), Turbofix by United Technologies (Duval, Owczarski, Paulonis, \& Schaefer, 1978), and Liburdi powder metallurgy (LPM) by Liburdi Engineering. All of these processes use high and low melting components to limit the amount of melting point depressant and to help bridge large gaps. The use of WGB has increased substantially the repair of aeroengine components over the last decades because these parts have become increasingly more difficult to weld due to the complex designs and use of less weldable materials. Although most WGB braze repair is performed on noncritical stationary parts, the use of brazing on low stress regions of rotating components is on the rise.

Transient liquid phase (TLP) bonding, a derivative of high temperature fluxless vacuum brazing, is widely used for post-service repairs in aerospace. In essence, the TLP process utilizes the concept of isothermal solidification of the liquid forming braze alloy to prevent the formation of brittle inter-metallic phases that normally form during cooling from conventional brazing. TLP bonding can be used to join a variety of material system including nickel base superalloy, titanium, composite and ceramic materials.

\subsection{Heat treatment}

\subsubsection{Rejuvenation heat treatment}

Material degradation occurs in hot section components that run for long periods of time at high temperature and stress conditions. To reduce the life cycle costs of hot section components, rejuvenation heat treatment processes have been developed to reverse the observed metallurgical damage back to the condition of a pristine alloy. Rejuvenation has typically been employed as part of an overall repair workscope that includes the removal and replacement of coatings and repair of mechanical damage and wear.

Rejuvenation heat treatments involve solving and aging steps which return the microstructure back to its optimal morphology. Component microstructure is shown to be restored, and the following stress rupture life of rejuvenation is returned to a condition fit for continued service. Over the past few decades, rejuvenation heat treatment process has been developed to repair the material degradation that inevitably occurs during service. Initially applied to forged polycrystalline superalloys, the process has proven effective for cast polycrystalline alloys but has been a challenge for single crystal superalloys.

\subsubsection{Straightening heat treatment}

Deformation occurs in hot section components that work at high temperature and stress conditions like material degradation. The dimensions of the deformed components occasionally require adjustment (e.g., the trailing edge of nozzle guide vanes and compressor disks). This can be accomplished by straightening heat treatment (i.e., plastically deforming the material). It is best accomplished in the solution treated condition for maximum ductility. In addition to cracking, the strain introduced by straightening can lead to the formation of creep voids and lowered creep properties during annealing. Therefore, the deformation mechanism, heat treatment regime, material properties and precautionary measures should be taken into account during making the straightening scheme.

\subsection{Coating \& plating}

In general, the environmentally induced coating or plating failure modes include high-temperature oxidation, hot corrosion, mechanical distress, solid-state diffusion, spallation and thermal-mechanical fatigue cracking. The remanufacture of coated or plated components requires that old spent coating or plating be removed from the parts and the new coating or plating be prepared after the defects of substrates have been repaired.

Coatings or plantings are typically stripped in strong, often heated mineral acid $\left(\mathrm{HCl}, \mathrm{HNO}_{3}\right.$, etc.) mixtures which react with the coatings or plating. The acid compositions are designed and controlled to selectively remove coating or plating without attacking the substrate alloy. In addition to chemical cleaning, water jet cleaning, fluorine-ions cleaning and salt bath cleaning are also used to remove the coatings or plating.

The plating is typically prepared by using electro-plating processes such as silver plating, nickel plating and copper plating. Although many types of coatings are currently in use, they generally fall into one of three types: Aluminide, chromide, and MCrAlY. A wide variety of processes are used to apply coatings, although they rely on one of the three general methods: Physical vapor deposition (PVD), chemical vapor deposition (CVD), and thermal spray. Diffusion coatings are well bonded to the substrate but have limited composition flexibility. Three primary methods for producing diffusion coatings are pack cementation, out-of-pack cementation, and chemical vapor deposition. Overlay coatings are typically well bonded and have a broad composition flexibility. Three primary methods for producing overlay coatings are physical vapor deposition, sputtering, and thermal spray. Thermal barrier coatings (TBCs) are overlay coatings and are designed to provide a thermally insulating layer between the gas and the component. TBCs consist of a layered structure of $\mathrm{Y}_{2} \mathrm{O}_{3}$ stabilized $\mathrm{Zr}_{2} \mathrm{O}_{3}$ over an oxidized bond coat of MCrAlY. TBCs are deposited using thermal 
spray (Ar shrouded plasma (APS) or low pressure plasma (LPPS)) or electron beam physical vapor deposition (EBPVD).

In addition, wear control coatings and compressor coatings must be mentioned. Two groups of coatings are used for wear control: Soft alloy compositions are used for fretting wear and abradable materials are used for clearance control. Both types of coating are applied by thermal spray. Coatings are used on compressor airfoils to provide increased resistance to corrosion and particle erosion. Currently three groups of coatings are in use: Filled paints (e.g., Sermaloy W) for corrosion, thick hard coatings (e.g., thermal spray $\mathrm{WC} \& \mathrm{CrC}$ ) and thin super-hard coatings (e.g., TiN and DLC (Diamond-like carbon) coating) for erosion.

\subsection{Surface strengthening}

It has long been known that the surface composition or structure or stress can be altered by the use of energy or particle beams and heat treatment. Surface strengthening refers to modify the surface integrity of the materials to improve the service behavior of fatigue, wear, and stress corrosion. Due to the stress relaxation effect, the surface strengthening processes are often used to induce compressive stresses in the exposed surface layers and restore and reinforce the required stress status and surface quality of the component during remanufacturing.

The common surface strengthening processes include shot peening, rolling compaction, cold extrusion, and ultrasonic shock peening. In recent years, as an innovative and promising technique, laser shock peening (LSP) has been successfully applied to improve fatigue performance of metallic components. The key beneficial characteristics after LSP treatment is the presence of compressive residual stresses beneath the treated surface of metallic materials, mechanically produced by high magnitude shock waves induced by a high-energy laser pulse.

\section{Aeroengine remanufacturing system}

An organizational system can be considered a set of dynamic and interdependent parts and functions with shared objectives. According to the authors' opinion and from a technology perspective, the aeroengine remanufacturing system is a complex technology cluster with a framework of "Failure analysis + Pre-processing + Remanufacturing + Life evaluation and prediction + Technology qualification and validation + Quality control and testing". As shown in Figure 4, its main elements and subelements are listed below:

- Element 1: Failure analysis

- Element 2: Pre-processing

O Sub-element 2.1: Cleaning

○ Sub-element 2.2: Coating and plating removal

- Element 3: Remanufacturing

O Sub-element 3.1: Surface integrity remanufacturing

O Sub-element 3.2: Design change and performance improvement

- Element 4: Life assessment and extension

O Sub-element 4.1: Life assessment

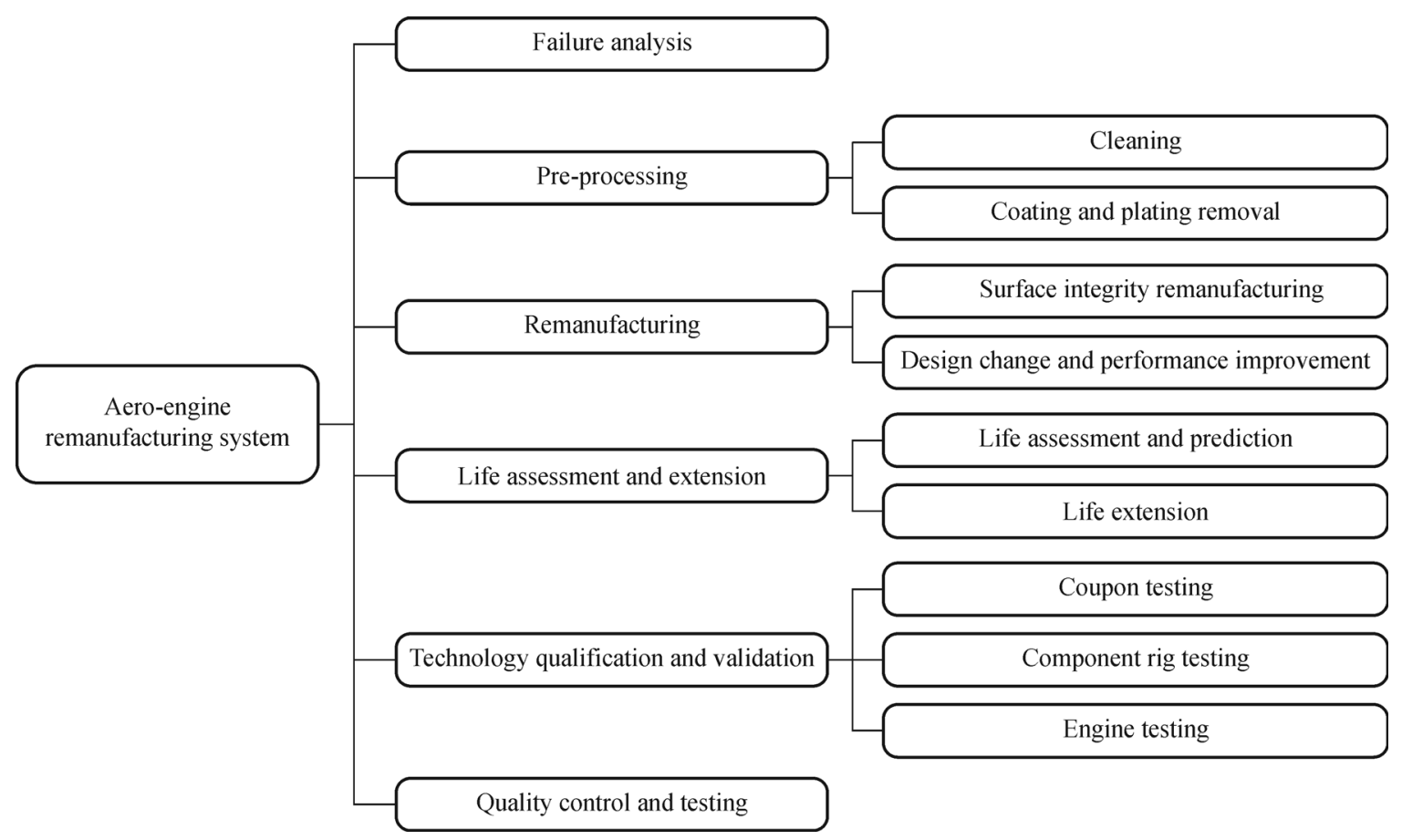

Figure 4. The framework of aeroengine remanufacturing system. 
Sub-element 4.2: Life extension

- Element 5: Technology qualification and validation

O Sub-element 5.1: Coupon testing

O Sub-element 5.2: Component rig testing

$\bigcirc$ Sub-element 5.3: Engine testing

- Element 6: Quality control and testing

The aeroengine remanufacturing system begins with the failure analysis. Failure analysis is the fundamental of remanufacturing and its task is to investigate the root cause of failure, and to get a thorough understanding of the failure mechanism, and to make a feasible, reliable and economic remanufacturing plan. The system is followed by preprocessing. It cleans the dirt, carbon deposit, oxide and corrosion, removes the old spent coating and plating, and makes preparations for the next step-Remanufacturing. Remanufacturing has two aspects: Surface integrity remanufacturing and design deficiency correction and performance improvement. As for surface integrity remanufacturing, the surface damages are repaired and the surface integrity are refreshed or improved by using remanufacturing technologies such as welding, brazing and thermal spray. As for design change and performance improvement, measures should be taken to improve the reliability and performance of the components. The next step is life assessment and extension. It is necessary to develop service-life assessment methodologies and refurbishment technologies to restore material properties and maximize and extend the safe service lives of aeroengine components. Technology qualification and validation is carried out after life assessment and extension. There are three qualification levels used to ensure the technology readiness and the safety and reliability of the components: Coupon level, component rig level and engine level. At coupon level, the microstructure, mechanical and surface properties are tested; at component rig level, the vibration, thermal fatigue properties as well as over-temperature testing and over-speed testing are evaluated; at engine level, accelerated engine test or block test are performed to provide a foundation for reliability, safety and durability. The final step is quality control and testing. Advanced testing technologies and quality control measures should be taken to ensure the surface quality, surface integrity and working performance.

\section{Applications}

According to the statistics, crack, oxidation, corrosion, wear, deformation, and materials degradation are the common surface damages during the service period of aeroengine components. These surface damages have been repaired and the surface integrity has been restored by using surface integrity remanufacturing technologies and basing on the proposed aeroengine remanufacturing system. Some typical application examples are list as follows:
- Example 1: Tip repair of compressor blade

Tip erosion and foreign object damage (FOD) are the common damages of compressor blade. The blade tip restoration is remanufactured by automated build-up welding/laser cladding, machining and benching to the original contour.

- Example 2: Patch repair of combustion liner

Due to the oxidation, hot corrosion, thermal fatigue, and local hot points of high-temperature gas, cracking and material missing are the common damages of combustion liner. Patch welding repair is used to repair the surface damages with large area of combustion liner.

- Example 3: Craze crack repair of nozzle guide vane Thermal fatigue and craze cracks of nozzle guide vane are remanufactured by wide gap brazing, solution heat treatments, TBC application, machining and benching to the original contour.

- Example 4: Tip repair of high-pressure turbine blade Thermal-mechanical fatigue cracks and tip erosion are the common damages of high-pressure turbine blade. The blade tip repair is remanufactured by laser cladding, rejuvenation heat treatment, TBC application, electrical discharge machining, machining and benching to the original contour.

Large numbers of aeroengine components have been successfully restored to original surface integrity and performance specification based on the engineering practices of surface integrity remanufacturing. Some applying effects and results of surface integrity remanufacturing are summarized as follows:

- Thousands of key components have been returned to service using surface integrity remanufacturing technologies, and the maintenance cost and spare parts of aeroengine are substantially reduced. Remanufacturing process preserves part of the raw materials and value added to the product, allowing company to increase the profitability and productivity. The average maintenance price is only $78 \%$ of the trade price in the same industry.

- The service life of aeroengine has been extended and the material potentialities have been exploited by restoration of the surface integrity using surface integrity remanufacturing technologies. The conservative service life and repair limits of some parts have been extended based on the thorough understanding of design specification, damage mechanism and surface integrity using remanufacturing technologies and technical qualification.

- The reliability and performance of the scrapped components are improved and reconstructed by remanufacturing techniques to obtain new service life. The maintenance cycle of aeroengine has been shortened by using remanufactured components. It is $40 \%$ of the cycle before using remanufacturing and is $30 \%$ of the specific cycle in the same industry.

- The aeroengine remanufacturing theory and system have been enriched and developed by surface integrity remanufacturing. Although the original surface integrity of 
the components is determined by design requirement and machining process, it should be restored and improved to meet the continued service requirements. The relation between the original surface integrity and the degraded surface integrity with the service life has been established.

\section{Conclusions}

With the rapid development of contemporary science and technology, more and more new materials (e.g., single crystal superalloy and composites), structures (e.g., blade integrated disk or ring) and processes (e.g., linear friction welding and laser shot peening) will be used in advanced high-performance aeroengine. Remanufacturing is a key element in realizing a sustainable society and surface integrity remanufacturing is a new direction of maintenance engineering. The future of the remanufacturing engineering appears positive with huge opportunities on the large quantity of end-of-life products in aerospace industry. However, there are considerable challenges in the remanufacturing applications. The advancement of surface integrity remanufacturing requires innovative remanufacturing process technologies and the state of the art technologies such as $3 \mathrm{D}$ printing and nano-scale processing will bring new vigor and vitality into remanufacturing. At the same time, combining with the existing technologies, it makes the remanufacturing components which are mainly used products reach zero scrap rate, and completely realizes the full recycling of resources.

\section{References}

ANSI-B211.1. (1986). American national standards on surface integrity. Washington, D.C: American National Standards Institute.

Axinte, D. A., \& Dewes, R. C. (2002). Surface integrity of hot work tool steel after high speed milling experimental data and empirical models. Journal of Materials Processing Technology, 127, 325-335.

Biller, S. (2011). Innovation in global manufacturing: Sustainability for business success. Como Lake: World Manufacturing Forum.

Demo, W., \& Ferrigno, S. J. (1992). Brazing method helps repair aircraft gas-turbine nozzles. Advanced Materials \& Processes, 141, 43-45.

Duval, D., Owczarski, W., Paulonis, D., \& Schaefer, R. (1978). Metallic filler material. U.S. Patent 4,073,639.

Ellison, K. A., Lowden, P., Liburdi, J., \& Boone, D. H. (1993). Repair joints in nickel-based superalloys with improved hot corrosion resistance. In: Proceedings of International Gas Turbine and Aeroengine Congress and Exposition. Cincinnati, 93-GT-247.

Field, M., \& Kahles, J. F. (1971). Review of surface integrity of machined components. Annals CIRP, 20, 153-163.

Griffiths, B. J. (2001). Manufacturing surface technology, surface integrity and functional performance. London: Manufacturing Engineering Modular Series, Penton Press. 